\title{
On abelian varieties associated with elliptic curves with complex multiplication
}

\author{
by \\ Tetsuo Nakamura (Sendai)
}

1. Introduction. Let $K$ be an imaginary quadratic field and $H$ the Hilbert class field of $K$. Let $E$ be an elliptic curve over $H$ with complex multiplication by $K$. We suppose that $E$ is a $K$-curve, that is, for each $\sigma \in \operatorname{Gal}(H / K), E^{\sigma}$ and $E$ are $H$-isogenous. We denote by $B=R_{H / K}(E)$ the abelian variety over $K$ which is obtained from $E$ by restriction of scalars. We will show that one of the following three cases holds (see Theorem 3 ):

(i) $B$ is a simple CM-type abelian variety over $K$.

(ii) $B$ is isogenous to a product $A \times \ldots \times A$ of a simple non-CM abelian variety $A$ such that $\operatorname{End}_{K} A \otimes \mathbb{Q}$ is commutative.

(iii) $B$ is isogenous to a product $A \times \ldots \times A$ of a simple non-CM abelian variety $A$ such that $\operatorname{End}_{K} A \otimes \mathbb{Q}$ is a division quaternion algebra.

Some examples of these cases are discussed in Section 4. In [B-Gr] and $[\mathrm{Gr}], \mathbb{Q}$-curves are treated under the assumption that the class number $h$ of $K$ is odd. Such a curve $E$ is a $K$-curve satisfying the condition: $E^{\tau}$ and $E$ are $H$-isogenous, where $\tau$ is the complex conjugation. In this case, it is shown that $B$ is a simple CM-type abelian variety (see $[\mathrm{Gr}], \S 15$ ).

Throughout the paper elliptic curves have complex multiplication by $K$ and the following notation is used:

- $K$ - an imaginary quadratic field,

- $C l(K)$ - the ideal class group of $K$,

- $h$ - the class number of $K$,

- $H$ - the Hilbert class field of $K$,

- $G(L / k)$ - the Galois group of a Galois extension $L / k$,

- $I_{k}, C_{k}$ - the idele group and the idele class group of a number field $k$,

- $R_{k / M}(E)$ - the abelian variety over $M$ which is obtained from an elliptic curve $E$ over $k$ by restriction of scalars to $M$.

2000 Mathematics Subject Classification: 11G05, 11G10, 11G15. 
2. $K$-curves and descending characters. Let $M$ be a finite extension of $K$ and $L$ be a finite Galois extension of $M$. Let $E$ be an elliptic curve over $L$ with complex multiplication by $K$. Denote by $J$ the set of $\sigma \in G(L / M)$ such that $E^{\sigma}$ is isogenous to $E$ over $L$. Clearly $J$ is a subgroup of $G(L / M)$ and we obtain (cf. [Gr], Chap. 4)

$$
\operatorname{dim}_{K} \operatorname{End}_{M} R_{L / M}(E) \otimes \mathbb{Q}=|J| .
$$

Definition. 1. If $J=G(L / M)$, then we call $E$ an $M$-curve.

2. Let $\psi_{E}$ be the Hecke character of an elliptic curve $E$ over $L$. If there exists a Hecke character $\phi$ of $M$ such that $\psi_{E}=\phi \circ N_{L / M}$, we say that $\psi_{E}$ descends to $M$ or simply that $E$ has an $M$-character $\phi$.

Remark. 1. The following fact is well known: $\psi_{E}$ descends to $M$ if and only if all the points of $E$ of finite order are rational over $M^{\mathrm{ab}} L$, where $M^{\mathrm{ab}}$ is the maximal abelian extension of $M$ (see [S1], Theorem 7.44).

2. For an elliptic curve $E$ over $H$ there exists an elliptic curve $E_{0}$ over $H$ such that $j_{E}=j_{E_{0}}$ and $E_{0}$ has a $K$-character (see [S2], Prop. 5, p. 525).

TheOREM 1. Let E, $L, M$ be as above and assume that $L$ is abelian over $M$. Then the following conditions are equivalent:

(i) $L\left(E_{\text {tors }}\right)$ is an abelian extension of $M$.

(ii) The abelian variety $B=R_{L / M}(E)$ has complex multiplication over $M$ in the sense that

$$
\operatorname{End}_{M} B \otimes \mathbb{Q} \cong \prod_{i=1}^{r} T_{i}
$$

where $T_{i}(i=1, \ldots, r)$ are $(C M)$ fields over $K$ such that

$$
\sum_{i}\left[T_{i}: K\right]=[L: M](=\operatorname{dim} B) .
$$

(iii) $E$ has an $M$-character.

In case $K=M$, the theorem is Théorème 4.1 in [G-Sch] and since our assertion is proved similarly, we omit its proof. If $L=H$, we have the following:

TheOREM 2. Let $M$ be a subfield of $H$ containing $K$. If $E$ is an elliptic curve over $H$ with an $M$-character, then $B=R_{H / M}(E)$ is a simple $C M$ type abelian variety over $M$, which means that $\operatorname{End}_{M} B \otimes \mathbb{Q}$ is a field over $K$ of degree $[H: M]$.

Proof. Since $R=\operatorname{End}_{K} B \otimes \mathbb{Q}$ is commutative by Theorem 1, it suffices to show that $R$ is a field of degree $[H: M]$ over $K$. If $M=K$ and $h$ is odd, the proof is given in $[\mathrm{Gr}]$, Chap. 4 . Our proof proceeds similarly. Let $Y$ be the subgroup of $C l(K)$ corresponding to $M$. Let $\mathfrak{a}$ be an integral ideal in $Y$. One can associate with $\mathfrak{a}$ an $M$-endomorphism $t(\mathfrak{a})$ of $B$ with the following 
property: If $\mathfrak{a}^{n} \sim 1$, then $t(\mathfrak{a})^{n} \in K$ and $\mathfrak{a}^{n}=\left(t(\mathfrak{a})^{n}\right)$ (see [Gr], Chap. 4). For a prime number $p$, let $Y_{p}$ be the $p$-Sylow subgroup of $Y$ and put $p^{r}=\left|Y_{p}\right|$. For a set of independent generators $\mathfrak{a}_{1}, \ldots, \mathfrak{a}_{s}$ for $Y_{p}$, let $X_{p}$ be the subgroup of $K^{\times} / K^{\times p^{r}}$ generated by $\left\{t\left(\mathfrak{a}_{i}\right)^{p^{r}} \mid 1 \leq i \leq s\right\}$. Then $Y_{p}$ is isomorphic to $X_{p}$. Let $T_{p}=K\left(\left\{t\left(\mathfrak{a}_{i}\right) \mid 1 \leq i \leq s\right\}\right)$. It suffices to show that $T_{p}$ is a field over $K$ of degree $p^{r}$, because we then have $\operatorname{dim}_{K} R=\operatorname{dim}_{K} \prod_{p} T_{p}$. Write $\mu\left(p^{r}\right)$ for the group of $p^{r}$ th roots of unity and put $K^{\prime}=K\left(\mu\left(p^{r}\right)\right)$. Now we use the following lemma which follows from [W], Lemma 13.27.

Lemma 1. If $p$ is odd, then $H^{1}\left(G\left(K^{\prime} / K\right), \mu\left(p^{r}\right)\right)=(0)$. If $p=2$, then $H^{1}\left(G\left(K^{\prime} / K(\sqrt{-1})\right), \mu\left(p^{r}\right)\right)=(0)$.

If $p$ is odd, then $K^{\times} / K^{\times p^{r}} \rightarrow K^{\prime \times} / K^{\prime \times p^{r}}$ is injective by Lemma 1 . Since $K^{\prime} T_{p}$ is a Kummer extension of $K^{\prime}$ corresponding to the subgroup $X_{p}$, it follows that $T_{p}$ is a field over $K$ of degree $p^{r}$. Now assume $p=2$. It suffices to consider the case when $h>1$ and the exponent of the group $Y_{2}$ is greater than 2. Then $K(\sqrt{-1})\left(=K_{1}\right.$ say $) \neq K$ and $\mu(4)=\mu\left(2^{r}\right)^{G\left(\bar{K} / K_{1}\right)}$. In the restriction inflation sequence

$$
0 \rightarrow H^{1}\left(G\left(K_{1} / K\right), \mu(4)\right)(\cong \mathbb{Z} / 2 \mathbb{Z}) \stackrel{i}{\rightarrow} K^{\times} / K^{\times 2^{r}} \rightarrow K_{1}^{\times} / K_{1}^{\times 2^{r}}
$$

the image of $i$ corresponds to the extension $K_{1} / K$. From this we see that $T_{2}$ is a field over $K$ of degree $2^{r}$, since $K_{1}^{\times} / K_{1}^{\times 2^{r}} \rightarrow K^{\prime \times} / K^{\prime \times 2^{r}}$ is injective by Lemma 1 . This completes the proof of Theorem 2 .

\section{The abelian variety $R_{H / K}(E)$}

Lemma 2. Let $M$ be a subfield of $H$ containing $K$. Let $E_{0}$ be an elliptic curve over $H$ with an $M$-character. Let $E$ be a twist of $E_{0}$ corresponding to a quadratic extension $k / H$. Then

(i) $E$ is an $M$-curve if and only if $k / M$ is Galois.

(ii) $E$ has an $M$-character if and only if $k / M$ is abelian.

Proof. Let $\psi_{0}, \psi$ be Hecke characters of $E_{0}, E$, respectively. Then by [Gr], Lemma 9.2.5, we have $\psi=\psi_{0} \cdot \chi$, where $\chi: I_{H} \rightarrow\{ \pm 1\}$ is the character associated with the extension $k / H$.

(i) $E$ is an $M$-curve if and only if $\psi^{\sigma}=\psi(\sigma \in G(H / M))$ (see [Gr], $\S 11)$. Our assertion follows from the equivalence of the following assertions:

(1) $\psi^{\sigma}=\psi(\sigma \in G(H / M))$.

(2) $\chi^{\sigma}=\chi(\sigma \in G(H / M))$.

(3) $\operatorname{Ker} \chi$ is $G(H / M)$-stable.

(4) $k / M$ is Galois.

(ii) If $k / M$ is abelian, our assertion is clear by Theorem 1 , since $R_{k / M}\left(E_{0}\right)$ $\cong R_{k / M}(E)$. Now assume that $\psi$ descends to $M$. Then $\psi=\phi \circ N_{H / M}$ and 
$\psi_{0}=\phi_{0} \circ N_{H / M}$, where $\phi$ and $\phi_{0}$ are characters of $I_{M}$. As $E_{0}$ and $E$ are isomorphic over $k, \phi$ and $\phi_{0}$ coincide on the norm subgroup $P_{k}=N_{k / M}\left(C_{k}\right)$ of $C_{M}$. Since $\chi$ is non-trivial, $\phi$ and $\phi_{0}$ differ on $P_{H}=N_{H / M}\left(C_{H}\right)\left(\supset P_{k}\right)$. This implies that $P_{H} \neq P_{k}$, which shows that $k / M$ is abelian.

TheORem 3. Let $E$ be a $K$-curve over $H$ and put $B=R_{H / K}(E)$ and $R=\operatorname{End}_{K} B \otimes \mathbb{Q}$. If $E$ has a $K$-character, $R$ is a field of degree $h$ over $K$. If $E$ has no $K$-characters, then the center $Z$ of $R$ is a field of degree $h_{0}$ over $K$ with $h=2^{2 m} h_{0}(m \geq 1)$ and one of the following two cases holds:

(i) $R \cong \mathrm{M}_{2^{m}}(Z)$. In this case, $B$ is isogenous over $K$ to a product of $A$ with itself $2^{m}$ times, where $A$ is $K$-simple, $2^{m} h_{0}$-dimensional and $Z=$ $\operatorname{End}_{K} A \otimes \mathbb{Q}$.

(ii) $R \cong \mathrm{M}_{2^{m-1}}(D)$, where $D$ is a division quaternion algebra over $Z$. In this case, $B$ is isogenous over $K$ to a product of $A$ with itself $2^{m-1}$ times, where $A$ is $K$-simple, $2^{m+1} h_{0}$-dimensional and $D=\operatorname{End}_{K} A \otimes \mathbb{Q}$.

Proof. Choose an elliptic curve $E_{0}$ over $H$ with a $K$-character such that $j_{E}=j_{E_{0}}$ (see Remark 2). If $E$ and $E_{0}$ are isomorphic over $H$, our assertion follows from Theorem 2. Assume that $E$ and $E_{0}$ are not isomorphic over $H$. Since it suffices to consider the case $h>1$, there exists a unique quadratic extension $k$ of $H$ such that $E$ and $E_{0}$ are isomorphic over $k$. Then $k / K$ is Galois by Lemma 2 and we have an exact sequence

$$
1 \rightarrow G(k / H)(\cong\{ \pm 1\}) \rightarrow G(k / K) \rightarrow G(H / K)(\cong C l(K)) \rightarrow 1 .
$$

Lemma 3. Let $C$ be the center of $G=G(k / K)$. Then $C$ contains $G(k / H)$ and $G / C$ is an elementary abelian group of order $2^{2 m}(m \geq 0)$ with $2 m \leq \operatorname{dim} C l(K) \otimes \mathbb{F}_{2}$. If $G$ is non-commutative, there exist $x_{1}, \ldots, x_{m}$, $y_{1}, \ldots, y_{m} \in G$ which induce a basis of $G / C$ and satisfy the following commutator relations:

$$
\left[x_{i}, y_{i}\right]=-1, \quad\left[x_{i}, x_{j}\right]=\left[y_{i}, y_{j}\right]=\left[x_{i}, y_{j}\right]=1 \quad(i \neq j) .
$$

Proof of Lemma 3. Since the commutator map

$$
G \times G \ni(x, y) \rightarrow[x, y] \in\{ \pm 1\}
$$

induces a non-degenerate alternating form on $G / C \times G / C$, our assertion follows easily.

If $E$ has a $K$-character, then $R=\operatorname{End}_{K}\left(R_{H / K}(E)\right) \otimes \mathbb{Q}$ is a field of degree $h$ over $K$ by Theorem 2. Now we assume that $E$ is a $K$-curve but has no $K$-characters, which means that $G$ is non-commutative by Lemma 2 . Let $m \geq 1$ be as in Lemma 3 and put $h_{0}=h / 2^{2 m}=|C /\{ \pm 1\}|$. Write $M_{0}$ and $M_{i}$ for the subfields of $H$ corresponding to $C$ and $\left\langle x_{i}, y_{i}, C\right\rangle$, respectively. As $G\left(k / M_{0}\right)=C$ is commutative, we see that $E$ has an $M_{0}$-character by Lemma 2 and $Z=\operatorname{End}_{M_{0}}\left(R_{H / M_{0}}(E)\right) \otimes \mathbb{Q}$ is a field over $K$ of degree $h_{0}$ 
by Theorem 2 . On the other hand as $G\left(k / M_{i}\right)$ is non-commutative, $E$ has no $M_{i}$-characters by Lemma 2 . Then by taking $L=H$ in Theorem 1 , we see that $D_{i}=\operatorname{End}_{M_{i}}\left(R_{H / M_{i}}(E)\right) \otimes \mathbb{Q}$ is not a direct product of fields. As $D_{i}$ is semisimple, this means that $D_{i}$ is a non-commutative subring of $R$ containing $Z$. By the map $G \rightarrow G(H / K) \cong C l(K), x_{i}$ and $y_{i}$ determine elements of $C l(K)$ and as in the proof of Theorem 2, they correspond to elements $s, t$ of $D_{i}$. We see that $D_{i}=Z[s, t]$ and $s^{2}, t^{2} \in Z$. According to $[\mathrm{Gr}]$, p. 47 , st and $t s$ differ by a root of unity in $K$; we get $s t=-t s$. Therefore $D_{i}$ is a quaternion algebra over $Z$. For $j \neq i$, we also have

$$
D_{j}=\operatorname{End}_{M_{j}}\left(R_{H / M_{j}}(E)\right) \otimes \mathbb{Q}=Z\left[s^{\prime}, t^{\prime}\right]
$$

where $s^{\prime}, t^{\prime}$ are elements of $D_{j}$ corresponding to $x_{j}, y_{j}$, respectively. Let $N$ be the subfield of $H$ corresponding to $\left\langle x_{i}, x_{j}, C\right\rangle$. Since $\left\langle x_{i}, x_{j}, C\right\rangle$ is commutative, $E$ has an $N$-character by Lemma 2 , so that $D^{\prime}=\operatorname{End}_{N}\left(R_{H / N}(E)\right) \otimes \mathbb{Q}$ is a field by Theorem 2. As $s, s^{\prime} \in D^{\prime} \subset R$, we have $s s^{\prime}=s^{\prime} s$. The same arguments show that elements of $D_{i}$ commute with those of $D_{j}$. Consequently, $D_{i} \cdot D_{j}=D_{i} \otimes_{Z} D_{j}$ in $R$ and in particular

$$
R=D_{1} \otimes_{Z} \ldots \otimes_{Z} D_{m} .
$$

In the Brauer group, the class to which $R$ belongs is a product of quaternion algebras; this implies that $R \cong \mathrm{M}_{2^{m}}(Z)$ or $R \cong \mathrm{M}_{2^{m-1}}(D)$, where $D$ is a division quaternion algebra over $Z$. This completes the proof of Theorem 3 .

COROLlary 1. If the 2-Sylow subgroup of $\mathrm{Cl}(\mathrm{K})$ is cyclic, i.e., if the discriminant of $K$ is divisible by at most two distinct primes, then every $K$-curve over $H$ has a $K$-character.

Proof. The inequality $2 m \leq \operatorname{dim} C l(K) \otimes \mathbb{F}_{2}$ in Lemma 3 implies that $G(k / K)$ is commutative. Our assertion follows immediately from Lemma 2.

4. Examples. We are going to discuss examples which show that both cases (i) and (ii) of Theorem 3 are possible.

Let $p_{1}, p_{2}$ and $q$ be three rational primes such that

$$
p_{1} \equiv p_{2} \equiv 1 \bmod 4, \quad q \equiv 3 \bmod 4 .
$$

The imaginary quadratic field $K=\mathbb{Q}\left(\sqrt{-p_{1} p_{2} q}\right)$ has discriminant $-p_{1} p_{2} q$. Let $\mathfrak{q}$ be the prime ideal of $K$ with $\mathfrak{q}^{2}=(q)$ and $\left(\frac{\alpha}{\mathfrak{q}}\right)$ denote the quadratic residue symbol mod $\mathfrak{q}$. Let $\phi_{0}$ be a Hecke character of $K$ such that for any principal ideal $(\alpha)$ of $K$ prime to $\mathfrak{q}$,

$$
\phi_{0}((\alpha))=\left(\frac{\alpha}{\mathfrak{q}}\right) \alpha .
$$

There are $h$ such characters (see [S2], p. 527, Example 3). We assume that $(*)$ the 2 -Sylow subgroup of $C l(K)$ is isomorphic to $\mathbb{Z} / 2 \mathbb{Z} \times \mathbb{Z} / 2 \mathbb{Z}$. 
Let $K_{0}$ be the subfield of $H$ over $K$ such that $G\left(H / K_{0}\right) \cong \mathbb{Z} / 2 \mathbb{Z} \times \mathbb{Z} / 2 \mathbb{Z}$ and put $K_{i}=K_{0}\left(\sqrt{p_{i}}\right)(i=1,2)$. Let $k$ be a quadratic extension of $H$ such that $k / K$ is Galois with non-commutative Galois group. Then $G\left(k / K_{0}\right)$ is of order 8 and is isomorphic to either the quaternion group or the dihedral group. Let $E_{0}$ be an elliptic curve over $H$ which corresponds to the Hecke character $\psi_{0}=\phi_{0} \circ N_{H / K}$. We write $E$ for a twist of $E_{0}$ with respect to $k / H$, so that the Hecke character of $E$ over $H$ is $\psi=\psi_{0} \cdot \chi$, where $\chi$ is the character defined as in the proof of Lemma 2. If we put $D=\operatorname{End}_{K_{0}}\left(R_{H / K_{0}}(E)\right) \otimes \mathbb{Q}$, then we see that

$$
R=\operatorname{End}_{K}\left(R_{H / K}(E)\right) \otimes \mathbb{Q}=Z \otimes_{K} D,
$$

where $Z$ is the center of $R$. For the prime ideal $\mathfrak{p}_{i}$ of $K$ with $\mathfrak{p}_{i}^{2}=\left(p_{i}\right)(i=$ $1,2)$, choose prime ideals $\mathfrak{l}_{i}$ of $K$ such that $\mathfrak{p}_{i}$ and $\mathfrak{l}_{i}$ belong to the same class in $C l(K)$ and the $\mathfrak{l}_{i}$ are unramified in $k / K$. Let $\mathfrak{L}_{1}$ be a prime ideal of $H$ lying over $\mathfrak{l}_{1}$. The decomposition field $Z_{1}$ of $\mathfrak{L}_{1}$ is of index 2 in $H$. As $k / Z_{1}$ is abelian, there exists a $Z_{1}$-character $\psi_{1}$ such that $\psi=\psi_{1} \circ N_{H / Z_{1}}$. Let $\mathcal{L}_{1}$ be the restriction of $\mathfrak{L}_{1}$ to $Z_{1}$. Then $\psi\left(\mathfrak{L}_{1}\right)=\psi_{1}\left(\mathcal{L}_{1}^{2}\right)$ and

$$
\psi\left(\mathfrak{L}_{1}\right)=\psi_{0}\left(\mathfrak{L}_{1}\right) \chi\left(\mathfrak{L}_{1}\right)=\phi_{0}\left(\mathfrak{l}_{1}^{2}\right) \chi\left(\mathfrak{L}_{1}\right)
$$

where $\chi\left(\mathfrak{L}_{1}\right)= \pm 1$ and $\phi_{0}\left(\mathfrak{l}_{1}^{2}\right)=\left(\frac{p_{1}}{q}\right) p_{1} a_{1}^{2}$ with $\mathfrak{l}_{1}=a_{1} \mathfrak{p}_{1}\left(a_{1} \in K^{\times}\right)$. Now let $\psi_{1}\left(\mathcal{L}_{1}\right)$ be an element of $\operatorname{End}_{Z_{1}}\left(R_{H / Z_{1}}(E)\right) \subset D$ satisfying $\psi_{1}\left(\mathcal{L}_{1}\right)^{2}=\psi\left(\mathfrak{L}_{1}\right)$. A similar argument also holds for $\mathfrak{l}_{2}$. Therefore $D$ is a quaternion algebra over $K$ generated by $t_{1}$ and $t_{2}$ with $t_{i}^{2}=\widehat{p}_{i}= \pm p_{i}(i=1,2)$ and $t_{1} t_{2}=-t_{2} t_{1}$. This implies that the splitting of $D$ is completely determined by the Hilbert norm residue symbol $\left(\frac{\widehat{p}_{1}, \widehat{p}_{2}}{\mathfrak{p}}\right)$. We easily get $\left(\frac{\widehat{p}_{1}, \widehat{p}_{2}}{\mathfrak{p}}\right)=1$ for a prime ideal $\mathfrak{p}$ of $K$ prime to 2 . Therefore if 2 does not split in $K$, we obtain $D \cong \mathrm{M}_{2}(K)$ by the product formula of the norm residue symbol. From now on we suppose that 2 splits in $K$. Let $\mathfrak{p}$ be a prime ideal of $K$ over 2 . We seek a condition for $\left(\frac{\widehat{p}_{1}, \widehat{p}_{2}}{\mathfrak{p}}\right)=-1$. Since the localization of $K$ with respect to $\mathfrak{p}$ is $\mathbb{Q}_{2}$, we have $\left(\frac{\widehat{p}_{1}, \widehat{p}_{2}}{\mathfrak{p}}\right)=-1$ if and only if $\widehat{p}_{i}=-p_{i}(i=1,2)$.

1) If $G\left(k / K_{0}\right)$ is the quaternion group, then the $G\left(k / K_{i}\right)$ are cyclic and this implies $\chi\left(\mathfrak{L}_{i}\right)=-1(i=1,2)$. Therefore if $\widehat{p}_{i}=-p_{i}$, then $\left(\frac{p_{i}}{q}\right)=1(i=$ $1,2)$, which contradicts the assumption $(*)$ (see $[\mathrm{R}-\mathrm{R}]$ ).

2) If $G\left(k / K_{0}\right)$ is dihedral, then $G\left(k / K_{0}\right)$ has a unique cyclic subgroup of order 4. Assume that the $G\left(k / K_{i}\right)(i=1,2)$ are not cyclic. Then we have $\chi\left(\mathfrak{L}_{i}\right)=1$. Consequently, $\left(\frac{\widehat{\underline{p}}_{1}, \widehat{p}_{2}}{\mathfrak{p}}\right)=-1$ if and only if $\left(\frac{p_{1}}{q}\right)=\left(\frac{p_{2}}{q}\right)=-1$.

Since $\left(\frac{p_{1}, p_{2}}{\mathfrak{p}}\right)=1$ for all places $\mathfrak{p}$ of $K$, there exist $a, b, c(\neq 0)$ in $K$ satisfying

$$
a^{2}=p_{1} b^{2}+p_{2} c^{2} .
$$

Put $x=\sqrt{a+b \sqrt{p_{1}}}$ and $k=H(x)$. Then $k / K_{0}$ is Galois, $G\left(k / K_{0}\right)$ is dihedral and $G\left(k / K_{0}\left(\sqrt{p_{i}}\right)\right)(i=1,2)$ is not cyclic (cf. [Se], 1.2). For exam- 
ple, take $p_{1}=5, p_{2}=17, q=3$. Then $h=12$ and 2 splits in $K$. Since $\left(\frac{p_{i}}{q}\right)=-1(i=1,2)$, we see that $R$ is a division quaternion algebra over a field $Z$ of degree 3 over $K$.

\section{References}

[B-Gr] J. P. Buhler and B. H. Gross, Arithmetic on elliptic curves with complex multiplication. II, Invent. Math. 79 (1985), 11-29.

[G-Sch] C. Goldstein et N. Schappacher, Séries d'Eisenstein et fonctions $L$ de courbes elliptiques à multiplication complexe, J. Reine Angew. Math. 327 (1981), 184218.

[Gr] B. H. Gross, Arithmetic on Elliptic Curves with Complex Multiplication, Lecture Notes in Math. 776, Springer, 1980.

[R-R] L. Rédei und H. Reichardt, Die Anzahl der durch 4 teilbaren Invarianten der Klassengruppe eines beliebigen quadratischen Zahlkörpers, J. Reine Angew. Math. 170 (1934), 69-74.

[Se] J.-P. Serre, Topics in Galois Theory, Jones and Bartlett, Boston, 1993.

[S1] G. Shimura, Introduction to the Arithmetic Theory of Automorphic Functions, Iwanami Shoten and Princeton Univ. Press, 1971.

[S2] - On the zeta function of an abelian variety with complex multiplication, Ann. of Math. 94 (1971), 504-533.

[W] L. C. Washington, Introduction to Cyclotomic Fields, Springer, New York, 1980.

Mathematical Institute

Tohoku University

Sendai 980-8578, Japan

E-mail: nakamura@math.tohoku.ac.jp

Received on 28.5.1999

and in revised form on 3.7.2000 